\title{
Implicit memory for novel associations between pictures: effects of stimulus unitization and aging
}

\author{
Irene P. Kan - Margaret M. Keane • Elizabeth Martin • \\ Elizabeth J. Parks-Stamm • Lindsay Lewis • \\ Mieke Verfaellie
}

Published online: 1 February 2011

(C) Psychonomic Society, Inc. (outside the USA) 2011

\begin{abstract}
Studies of implicit memory for novel associations have focused primarily on verbal materials and have highlighted the contribution of conceptually unitized representations to such priming. Using pictorial stimuli in a perceptual identification task, we examined whether new association priming can occur at a purely perceptual level. By manipulating the spatial contiguity of stimuli, we also evaluated whether such priming requires the creation of perceptually unitized representations. Finally, we examined the status of such priming in aging. In Experiment 1, we found that spatial contiguity of stimuli is not necessary for novel pictorial association priming to emerge, although such contiguity does enhance the magnitude of associative priming. In Experiment 2, we found that new association priming is age invariant, regardless of spatial contiguity. In Experiment 3, we provide additional evidence that pictorial association priming is perceptually based. These findings expand the scope and delineate the conditions of novel association priming and inform theories about the nature of implicit memory for new associations.
\end{abstract}

I. P. Kan $(\square)$

Department of Psychology, Villanova University,

800 Lancaster Avenue,

Villanova, PA 19085, USA

e-mail: irene.kan@villanova.edu

I. P. Kan • M. M. Keane • E. Martin • E. J. Parks-Stamm •

M. Verfaellie

Memory Disorders Research Center, Boston VA Healthcare

System and Boston University School of Medicine,

Boston, MA, USA

M. M. Keane $\cdot$ L. Lewis

Department of Psychology, Wellesley College,

Wellesley, MA, USA
Keywords Implicit memory $\cdot$ Novel associations · Priming · Aging

The ability to link together different elements of an experience is a fundamental aspect of memory, and the study of associative memory has a long and prominent history (e.g., Ebbinghaus, 1885). In recent years, the scope of investigations into associative memory has been broadened by the use of repetition priming paradigms, which enable an examination of the implicit memory mechanisms that may support memory for novel associations. Such studies have been important not only because they speak to the processes that support associative memory, but also because they elucidate the nature of the representations that support priming effects more generally (for reviews, see Keane \& Verfaellie, 2003; Uttl, Graf, \& Cosentino 2003).

In studies of priming for novel associations, subjects are typically exposed to pairs of items in a study phase and, in a later test phase, are reexposed to different combinations of previously studied items in the context of an unrelated task (e.g., lexical decision, stem completion, perceptual identification). In the test phase, subjects are typically presented with pairs consisting of elements that were seen together during the study phase (old pairs), pairs consisting of elements both of which were seen during the study phase but as part of separate pairs (recombined pairs), and pairs consisting of elements not seen during the study phase (new pairs). Enhanced test performance in the old, as compared with the recombined, condition (such as higher accuracy or shorter response time) reflects successful implicit memory (or priming) for the novel association established in the study phase.

To date, studies of novel association priming have focused almost exclusively on factors that promote associative priming in the verbal domain (e.g., Gabrieli, Keane, Zarella, \& Poldrack 1997; Goshen-Gottstein, Moscovitch, \& Melo 2000; Keane, 
Gabrieli, Noland, \& McNealy 1995; Moscovitch, Winocur, \& McLachlan 1986; for a review, see Zeelenberg, Pecher, \& Raaijmakers 2003). A number of manipulations have been identified to promote associative priming, including repetition (e.g., Howard, Heisey, \& Shaw 1986; Light, Kennison, Prull, La Voie, \& Zuellig 1996; Musen \& Squire, 1993a, 1993b), meaning elaboration (e.g., Graf \& Schacter, 1985; Howard, Fry, \& Brune 1991; Light, La Voie, \& Kennison 1995; Micco \& Masson, 1991; Pecher \& Raaijmakers, 2004; Reingold \& Goshen-Gottstein, 1996; Schacter \& Graf, 1986), and physical integration (e.g., Light et al., 1996; Musen \& Squire, 1993b).

For instance, Graf and Schacter (1985) found that meaning elaboration may be crucial to associative priming. In that study, subjects were presented with unrelated word pairs (e.g., balance-chair), and the extent of elaborative encoding was manipulated at study via task instructions. The authors observed significant associative priming in the elaborative encoding condition (sentence generation), but not in the nonelaborative encoding condition (vowel comparison of word pairs). The authors suggested that elaborative encoding facilitates associative priming because it allows the establishment of a unitized representation at encoding. A unitized representation results from the successful integration of distinct constituents into a single, coherent entity; once items are unitized at encoding, reactivation of any part of the unitized representation at subsequent retrieval will facilitate activation of the entire representation (e.g., Ceraso, 1985; Graf \& Schacter, 1989; Mandler, 1982; Mayes, Montaldi, \& Migo 2007; Musen, Szerlip, \& Szerlip 1999; Quamme, Yonelinas, \& Norman 2007), yielding associative priming. As such, unitization is conceived of as the glue that binds together the distinct constituents.

Reflecting the distinction between conceptual and perceptual associative priming (Graf \& Schacter, 1989), it is assumed that unitization may be either conceptually or perceptually mediated. While conceptual unitization may be achieved by elaboration of meaningful links between constituent elements, perceptual unitization may be achieved by presentation of constituent elements in close spatial proximity. For example, Light et al., (1996, Experiment 2) examined associative priming in a speeded reading task in which the stimuli were single-syllable words that were conjoined to form novel words (e.g., fishdust, townplant). Subjects showed associative priming: They were faster to read repeated old stimuli (e.g., fishdust) than repeated recombined stimuli (fishplant). Although associative priming in this task may have been the product of perceptual unitization of the conjoined words, one cannot rule out the possibility that the spatial proximity of the constituents promoted conceptual unitization of the word pairs, which, in turn, might allow associative priming to occur. For example, when shown the stimulus cowlamp, subjects might have spontaneously generated a new meaning for this novel word (e.g., a lamp that is shaped like a cow). This possibility is compatible with the notion that the verbal system is inherently relational, since its primary function is to link together separate constituents to create meaning (Sadoski \& Paivio, 2001). Thus, even under conditions thought to promote perceptual unitization (spatial proximity), novel association priming may nonetheless reflect the creation of a conceptually unitized representation, because top-down semantic effects may be unavoidable with verbal stimuli. As such, findings from the verbal domain indicate that conceptual unitization may play an important role in novel association priming, and they leave open the question as to the role of perceptual unitization in associative priming.

Studies examining associative priming in the pictorial domain may provide a different window into the mechanisms that promote implicit memory for novel associations: Because the spatial conjoining of two pictorial stimuli does not automatically give rise to a new conceptual representation (e.g., presenting a picture of a cow and a lamp in physical contact does not suggest a novel concept that combines these elements), such studies allow an opportunity to examine whether perceptual unitization is sufficient for associative priming. Furthermore, by manipulating the ability to perceptually unitize pictorial stimuli, one can examine whether new association priming can be obtained even in the absence of any form of unitization.

There is limited evidence regarding the existence of novel association priming in the pictorial domain. To our knowledge, the only study that has examined novel pictorial association priming comes from Dean and Young (1996, Experiment 6). In that experiment, subjects viewed pairs of line drawings of real objects, arranged in a top-bottom fashion. Half of the pairs consisted of two exemplars of the same object (same condition), and half of the pairs were composed of line drawings of two different objects (different condition). At study, subjects' task was to determine whether the two drawings portrayed the same object or two different objects. At test, individuals again performed a same/different judgment task. Associative priming could be evaluated only on different trials, since these were the only ones that involved establishment of a new association. Trials in the different condition included old, recombined, and new picture pairs. Associative priming would be indicated by a shorter response time to different-old pairs than to different-recombined pairs. No associative priming was observed in this task. ${ }^{1}$ This

\footnotetext{
${ }^{1}$ It is worth noting that novel association priming has been observed in studies focusing on implicit memory for associations between a visual shape and a contextual feature (e.g., color; Ceraso, 1985; Chun \& Phelps, 1999; Musen et al., 1999). However, it is unclear whether associative priming in the nonverbal domain can be obtained when two distinct equivalent items are to be linked, as is the case in the verbal domain.
} 
finding is important because the failure to obtain associative priming in this pictorial task leaves open the possibility that priming of novel associations may occur only for elements that give rise to a conceptually unitized representation (as described above in the verbal domain). ${ }^{2}$

However, in Dean and Young's (1996) study, not only did the use of pictorial stimuli preclude the automatic establishment of conceptually unitized representations, but also the experimental conditions did not foster the establishment of perceptually unitized representations. Studies in the attention literature have highlighted the role of spatial proximity in stimulus processing and have demonstrated that as spatial separation between two items decreases, the likelihood of processing the items as a single unit increases (Treisman, Kahneman, \& Burkell 1983). Because the pictorial stimuli in Dean and Young's study were not presented in a spatially contiguous manner, they may not have yielded perceptually unitized representations. It is possible that priming of novel pictorial associations may occur if and only if the constituent elements are perceptually unitized.

Another feature of Dean and Young's (1996) study that may have contributed to a null effect is their use of a same/different judgment task. It is possible that any facilitation due to implicit memory for the studied association between two distinct pictures (i.e., old pairs) was countered by a disinclination to respond different to a studied pairing. That is, when confronted with an old pair at test, the nature of the same/different task required the subjects to counter the sense of fluency associated with an old pair with a negative (i.e., different) response. This circumstance may have selectively lengthened reaction times to old pairs and blunted a reaction time difference between the old and recombined conditions.

In the present study, we revisited the question as to whether it is possible to obtain associative priming for novel picture pairs, with the goal of elucidating the boundary conditions of novel association priming. We evaluated this possibility with a perceptual identification task that eliminated the potential response confound associated with the same/different task. Following Treisman et al. (1983), we operationalized perceptual unitization in terms of spatial contiguity of stimulus pairs; in the encoding phase, picture pairs were presented in either a spatially contiguous manner or a spatially separated manner. If we obtained new association priming in this task, it would suggest that new association priming can occur in the absence of conceptual unitization. Furthermore, if such priming was limited to the spatially contiguous condition, it

\footnotetext{
${ }^{2}$ Although there are examples of perceptually mediated associative priming in the verbal domain (Gabrieli et al., 1997; Goshen-Gottstein \& Moscovitch, 1995), the use of verbal stimuli in these tasks leaves open the possibility that an emergent conceptual association influences the associative priming effect.
}

would suggest that perceptual unitization is necessary for pictorial association priming and that Dean and Young's (1996) failure to obtain associative priming was due to a lack of perceptual unitization. Alternatively, if priming occurred in both the spatially contiguous and separated conditions, it would suggest that the establishment of a perceptually unitized representation is not necessary for new pictorial association priming and that Dean and Young's failure to obtain priming in their task was an artifact of the response requirement.

\section{Experiment 1: effects of spatial contiguity of stimuli on novel association priming between pictures}

Method

\section{Subjects}

Thirty-six college students participated in this study: Eighteen took part in the contiguous condition, and 18 in the separated condition. In accordance with the procedures of the Institutional Review Board, all individuals provided informed consent prior to testing. Subjects were paid for their time, and they were tested individually. Each session lasted approximately $25 \mathrm{~min}$.

\section{Design and materials}

Pair type (old, new, recombined) was manipulated within subjects, and spatial contiguity (contiguous, separated) was manipulated between subjects.

Contiguous condition The stimuli were 304 unique objects (e.g., peach, binoculars), and they were randomly paired to form 152 dyads that were photographed for presentation in the experiment. All the pairs were photographed against a light taupe background to maintain uniformity of stimulus presentation. The two objects were placed in such a way that they were in physical contact with each other (see Fig. 1). Item pairing was restricted such that the two items were neither semantically nor thematically related. Thirtytwo pairs were used in the practice phase, and the remaining 120 pairs served as critical stimuli to assess novel association priming. (These were the original pairings.) The 120 pairs were divided into two sets of 60 original pairs; one set was used for half of the subjects, and the other set for the other half. Each set of 60 original pairs was divided into three groups of 20 pairs. For each group of 20 , a list of recombined pairs was constructed by randomly re-pairing (and rephotographing) an object of one pair with another object from a different pair, keeping left/right item position and orientation constant. 
Fig. 1 Sample trial sequence in the contiguous condition. Photographs have been resized and changed to grayscale for illustration purposes only

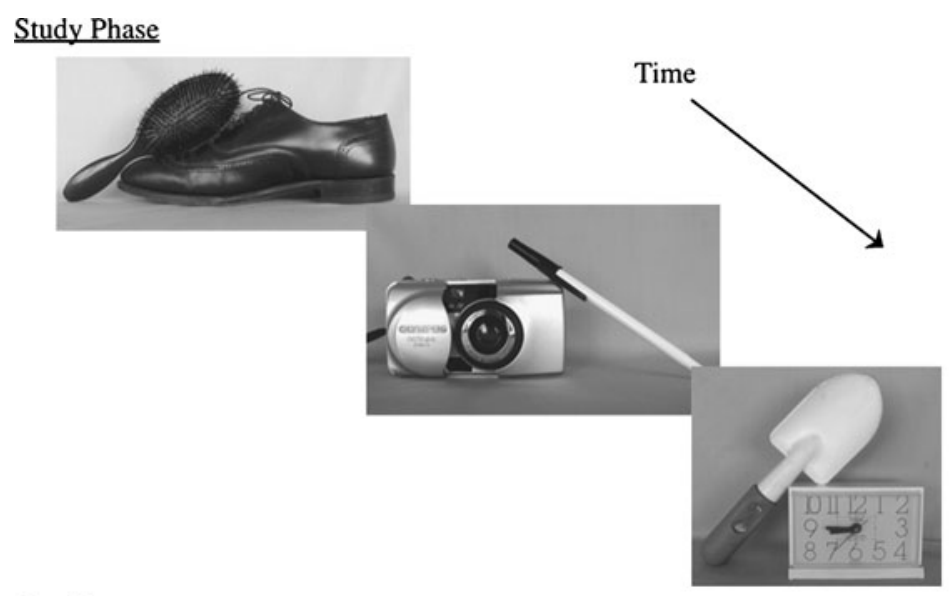

\section{Test Phase}

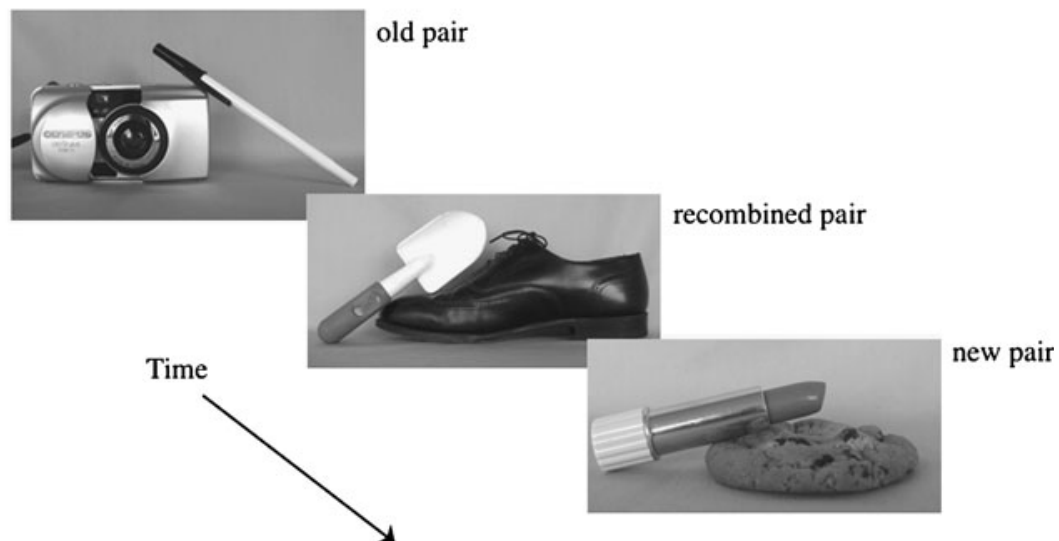

Separated condition A subset of items from the contiguous condition were used as stimuli in the separated condition. The stimuli were 184 unique objects that were randomly paired to form 92 dyads, and they were photographed against a light taupe background for presentation in the experiment. Objects were placed side by side, but not in physical contact (see Fig. 2). The same criteria as those described in the contiguous condition were used to create object pairings. Thirty-two pairs were used in a practice phase, and the remaining 60 original pairs were the critical stimuli used to assess priming of novel associations. These 60 original pairs were divided into three groups of 20 . For each group, a recombined list was created as described above. Of the 60 original pairs used in this condition, 58 were also used as original pairs in the contiguous condition.

\section{Procedure}

Each experimental session consisted of three phases: practice, study (object naming), and test (perceptual identification). From the subjects' perspective, the three parts were independent of each other. All the stimuli were presented using PsychLab stimulus presentation software on a Macintosh PowerBook computer.

Practice phase The goal of the practice phase was to familiarize subjects with the perceptual identification procedure. Each trial began with presentation of a fixation cross for $500 \mathrm{~ms}$. This was followed immediately by a photograph of a pair of objects that was flashed for $33 \mathrm{~ms}$ and then masked by a colorful geometric pattern that remained on the screen for $500 \mathrm{~ms}$. Individuals were instructed to identify the two objects in each photograph (by naming them aloud) and were encouraged to guess if they were unsure. A total of 32 unique photographs were shown.

Study phase (object naming) During the study phase, 40 photographs of object pairs were presented in full view on the computer screen, and individuals were asked to name aloud the two objects in each photograph. Each photograph remained on the screen until a response was made.

Test phase (perceptual identification) Each trial consisted of a brief (33-ms) presentation of a photograph, preceded by a fixation cross and followed by a mask, as described in the practice phase. Subjects saw the 60 original pairs, 
Fig. 2 Sample trial sequence in the separated condition. Photographs have been resized and changed to grayscale for illustration purposes only

\section{Study Phase}

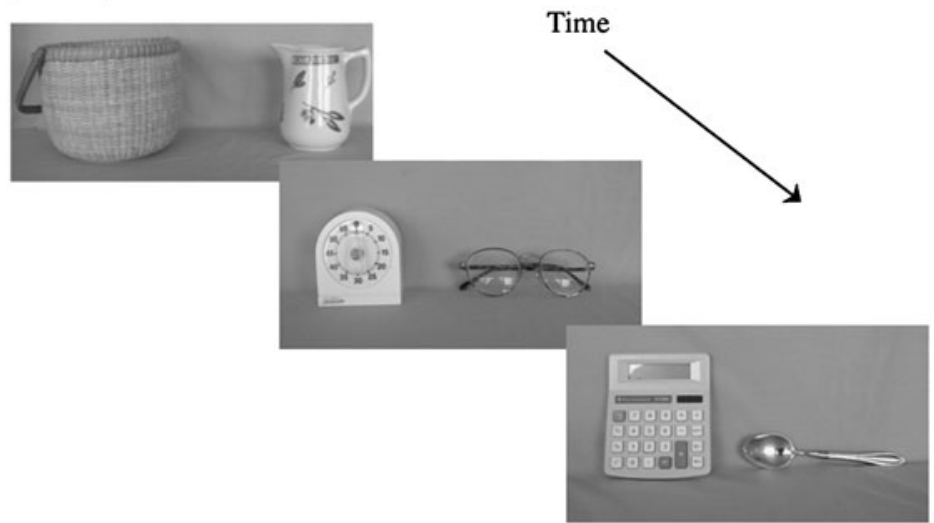

Test Phase

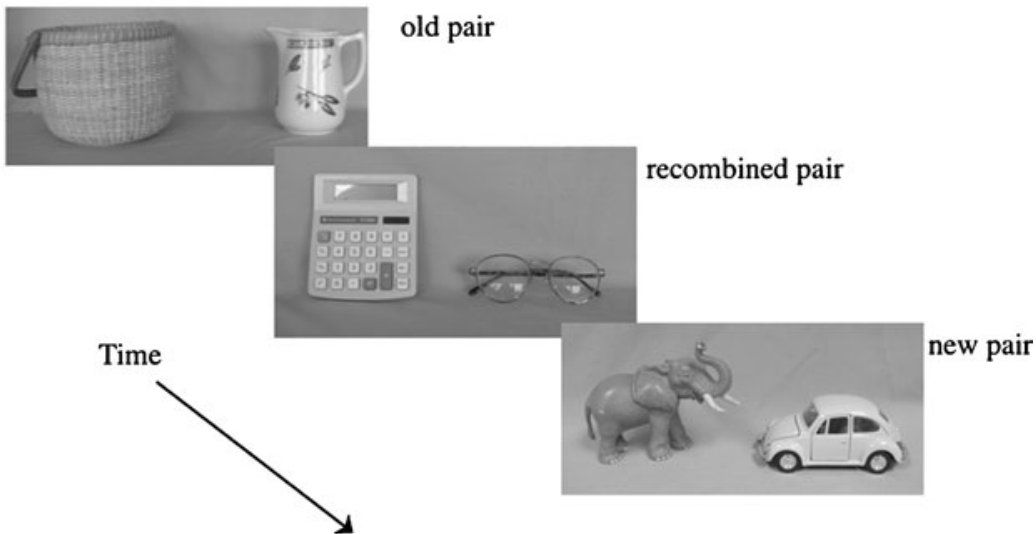

of which 20 had been seen in the study phase in the identical pairing (old condition), 20 had been seen in the recombined pairing (recombined condition), and 20 had not been seen (new condition). Thus, object pairs were always presented in their original configurations in the test phase, and experimental condition was defined by whether and how the pairs had been presented in the study phase. Subjects were instructed to identify the two objects in each photograph and were encouraged to guess if they were unsure. Across subjects, object pairs were counterbalanced such that each pair appeared equally often in the old, new, and recombined conditions.

\section{Results}

For each individual, percentage of accuracy of perceptual identification in each condition was calculated. A trial was considered correct if both items were named accurately (or with sufficient specificity to ensure adequate perception). Mean accuracy across subjects in each condition is summarized in Fig. 3.

To assess novel association priming and whether spatial contiguity modulated such priming, we compared accuracy in the test phase for old pairs with that for recombined pairs. We did so with a 2 (spatial contiguity: contiguous, separated) $\times 2$ (pair type: old, recombined) mixed analysis of variance (ANOVA). This analysis revealed a significant main effect of pair type, $F(1,34)=34.26, p<.001$, indicating better overall performance in the old condition

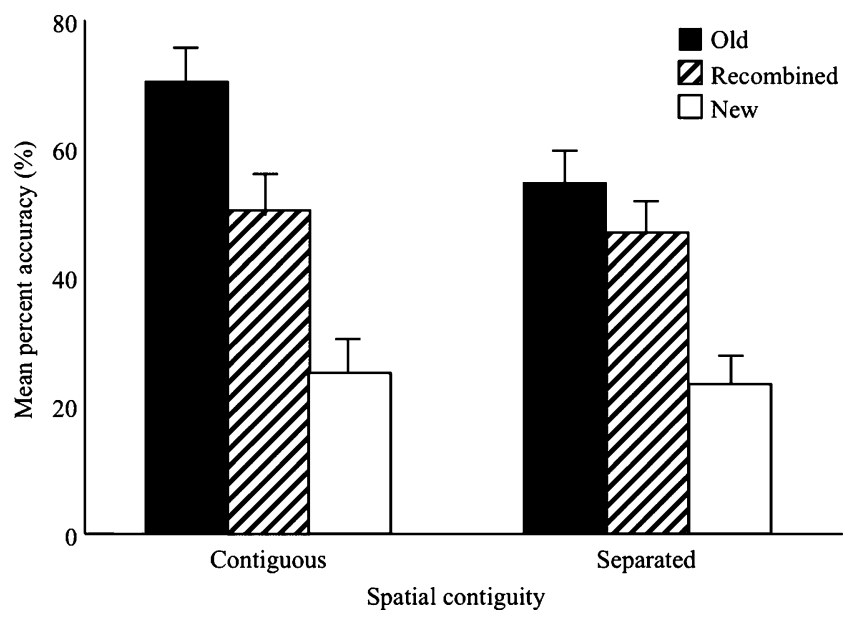

Fig. 3 Perceptual identification performance on old, recombined, and new trials in the contiguous and separated conditions (Experiment 1) 
$(M=62.5 \%, S D=18.4 \%)$ than in the recombined condition $(M=49.0 \%, S D=19.4 \%)$. We also found a marginal main effect of spatial contiguity, $F(1,34)=3.14, p=.09$, suggesting better performance in the contiguous condition $(M=60.7 \%, S D=17.8 \%)$ than in the separated condition $(M=50.8 \%, S D=15.9 \%)$ across the two pair types. Critically, we found a significant spatial contiguity $\times$ pair type interaction, $F(1,34)=6.55, p<.05$, indicating that the associative priming effect in the contiguous condition (mean associative priming $=19.4 \%$ ) was larger than that in the separated condition (mean associative priming $=7.6 \%$ ). ${ }^{3}$ Analysis of simple effects revealed that associative priming was significant in both the contiguous and the separated conditions (both $p \mathrm{~s}<.05$ ).

To assess item priming, we examined accuracy in the test phase for recombined pairs, as compared with new pairs. We did so with a 2 (spatial contiguity: contiguous, separated) $\times 2$ (pair type: recombined, new) mixed ANOVA. This analysis revealed a significant main effect of pair type, $F(1,34)=96.55, p<.001$, with higher accuracy in the recombined condition than in the new condition $(M=24.2 \%, S D=15.6 \%)$. No other effects were significant, with all $p \mathrm{~s}>.50$.

\section{Discussion}

In Experiment 1, we found novel association priming for picture pairs, both when stimuli were spatially contiguous and when they were spatially separated, although priming was greater in the former than in the latter condition. These findings with pictorial stimuli suggest that novel association priming can occur even when the paired constituents do not give rise to a conceptual representation of the association (i.e., when the new association is represented at a purely perceptual level). The finding that associative priming was significant even when items were spatially separated suggests that such priming does not require

\footnotetext{
3 The interpretation of the significant spatial contiguity $\times$ pair type interaction may, however, be limited by the fact that baseline (i.e., recombined) performance was numerically higher in the contiguous condition $(M=51.0 \%)$ than in the separated condition $(M=46.9 \%)$, although the difference was not statistically significant, $t<1$. To address this potential issue, we conducted a 2 (spatial contiguity: contiguous, separated) $\times 2$ (pair type: old, recombined) mixed ANOVA on accuracy, based on data from a subset of subjects whose recombined performance was matched (contiguous, $n=16$, mean recombined performance $=47.1 \%$; separated, $n=17$, mean recombined performance $=48.5 \%$ ). The ANOVA revealed largely the same pattern, including a significant spatial contiguity $\times$ pair type interaction, $F(1,31)=8.35, p<.01$. Once again, these results indicate that although associative priming was significant in both spatial contiguity conditions (both $p \mathrm{~s}<.05$ in an analysis of simple effects), the associative priming effect in the contiguous condition (mean associative priming $=20.9 \%$ ) was larger than that in the separated condition (mean associative priming $=6.9 \%$ ).
}

perceptual unitization of the constituents. However, our data also indicate that perceptual unitization, made possible by presenting stimuli in a spatially contiguous manner, enhances associative priming. Finally, these findings suggest that Dean and Young's (1996) failure to obtain novel pictorial association priming was likely due to the use of a same/different paradigm.

Our findings elucidate the scope and nature of implicit memory for novel associations. These insights, however, depend on the assumption that the observed effects are indeed implicit in nature. Although the perceptual identification task is considered to be a relatively "pure" implicit task (e.g., Paller \& Mayes, 1994; Yang et al., 2003), one might question whether the observed associative priming could be a by-product of explicit memory: Recall of one item of the pair could lead to explicit retrieval of the other item in the pair, which would enhance performance in the old, as compared with the recombined, condition. Evidence from studies of amnesic individuals may be instructive in this regard. For instance, using the perceptual identification task with verbal stimuli, Gabrieli et al. (1997) found equivalent magnitude of associative priming in amnesics and healthy controls, suggesting that the task is implicit in nature. Otherwise, differential performance between amnesic patients and controls would have been observed. However, the generalizability of these findings from the verbal domain to the present paradigm remains unclear.

To evaluate the possibility of explicit contamination in the present study, we examined errors in the recombined condition. If individuals' performance were contaminated by explicit memory, we would expect to observe intrusions of the studied associates on incorrect recombined trials. Such errors, however, were very rare. On average, of the recombined errors made by subjects in the contiguous condition, $9.2 \%$ were intrusions $(0.89$ intrusion errors out of 9.7). Similarly, on average, of the recombined errors made by subjects in the separated condition, $4.7 \%$ were intrusions ( 0.50 intrusion errors out of 10.6). These findings provide no evidence for a contribution of explicit memory to associative priming.

In sum, the present findings demonstrate that novel pictorial association priming can be obtained. Although such priming occurred both when the stimuli were spatially contiguous and when they were spatially separated, spatial contiguity of stimuli at study did enhance the magnitude of priming. We postulate that spatial contiguity may facilitate the creation of a perceptually unitized representation in the pictorial domain, in a manner analogous to the effect of semantic elaboration on conceptual unitization in the verbal domain. Because associative priming in this task is enhanced by perceptual unitization, and because unitization has been cited as a particularly important factor for associative priming to occur in elderly individuals (see the 
discussion below), the present task provides a useful tool for understanding the status of novel association priming in aging. We examined this issue in Experiment 2.

\section{Experiment 2: effects of aging and spatial contiguity of stimuli on novel association priming between pictures}

It is well established that older adults have impaired explicit memory for novel associations (La Voie \& Light, 1994; Old \& Naveh-Benjamin, 2008; Rhodes, Castel, \& Jacoby 2008), but the status of implicit memory for novel associations is less clear (for reviews of aging effects on implicit memory in general, see Fleischman \& Gabrieli, 1998; Light, Prull, La Voie, \& Healy 2000; Mitchell, 1993; Parkin, 1993). In the verbal domain, some studies have reported that new association priming may not be as robust in older adults as in younger individuals. For instance, it has been suggested that encoding conditions that promote conceptual unitization (e.g., elaboration and repetition; Howard et al., 1991; Howard et al., 1986; Musen \& Squire, 1993b) may be more important for associative priming in older adults, although this has not always been observed (Light et al., 1996, 1995).

To our knowledge, no studies have examined the effects of aging on novel association priming for pictures. Here, we assess the status of such priming using the perceptual identification task employed in Experiment 1. We also evaluate whether, by analogy to the verbal domain, conditions that promote unitization are critical for obtaining associative priming in the pictorial domain in older individuals. If perceptual unitization is required for associative priming to emerge in older adults, older subjects should show normal associative priming only in the spatially contiguous condition in the present paradigm. Alternatively, if perceptual unitization is not necessary for new association priming in older individuals, they should show normal associative priming in both the spatially contiguous and spatially separated conditions.

\section{Method}

\section{Subjects}

Seventy-two individuals (36 younger and 36 older) participated in this study. The younger group consisted of college students who had a mean age of 19.5 years $(S D=1.5)$, and the older group had a mean age of 64.1 years $(S D=9.6)$ and a mean education level of 16 years $(S D=2.2)$. All the older adults had intact cognitive functioning, as evidenced by performance above 140 on the Dementia Rating Scale-2 (Mattis, 2008). Half of the individuals from each age group participated in the contiguous condition (18 younger, 18 older), and the other half took part in the separated condition. There was no difference in age between younger subjects in the contiguous condition (younger subjects, $=19.5$ years, $S D=$ 1.1 ) and those in the separated condition (younger subjects, $M=19.5$ years, $S D=1.9), t(34)<1$. Similarly, there was no age difference between older subjects in the contiguous condition (older subjects, $M=65.4$ years, $S D=9.0$ ) and those in the separated condition (older subjects, $M=$ 62.8 years, $S D=10.2), t(34)<1$. In accordance with the procedures of the Institutional Review Board, all individuals provided informed consent prior to testing, and they were paid for their participation.

\section{Design and materials}

The materials used in this experiment were identical to those described in Experiment 1. Pair type (old, new, recombined) was manipulated within subjects, and spatial contiguity (contiguous, separated) and age group (older, younger) were manipulated between subjects.

\section{Procedure}

Each experimental session consisted of three phases: threshold setting, study (object naming), and test (perceptual identification). All the stimuli were presented using PsychLab stimulus presentation software on a Macintosh PowerBook computer. Each session lasted approximately $25 \mathrm{~min}$.

Threshold setting Given the increased variability in perceptual speed in older adults (Schaie, 1989), it was important to determine an individualized perceptual threshold for each subject. By establishing an exposure duration at which individuals would achieve approximately $25 \%$ accuracy in perceptual identification, we hoped that this procedure would minimize baseline performance differences between the two age groups (i.e., performance differences in the new condition).

In the threshold-setting procedure, each trial began with presentation of a fixation cross for $500 \mathrm{~ms}$. This was followed immediately by a photograph of an object pair that was flashed briefly and then masked by a colorful geometric pattern that remained on the screen for $500 \mathrm{~ms}$. Subjects were instructed to name aloud the two objects in each photograph and were encouraged to guess if they were unsure. A total of 32 unique photographs, divided into four sets of 8 , were shown. For older subjects, the exposure duration for the first set of photographs was $183 \mathrm{~ms}$, and exposure duration for subsequent sets of stimuli was either increased or decreased as needed to more closely approximate $25 \%$ accuracy. Adjustments in stimulus duration were selected on the basis of the experimenter's judgment of how 
large a change in exposure duration was needed to achieve the needed change in performance but was constrained by the refresh rate of the computer monitor, which was $16.7 \mathrm{~ms}$. Thus, the exposure duration could be changed only in increments that were multiples of $16.7 \mathrm{~ms}$. This process was repeated 4 times, with the goal of determining the exposure duration necessary to elicit an accuracy of approximately $25 \%$. For younger subjects, the initial exposure duration was set at $50 \mathrm{~ms}$, and the same titration procedure was followed. These individually determined exposure durations were used in the subsequent test (i.e., perceptual identification) phase.

The procedures involved in the study and test phase were identical to those in Experiment 1. From the subjects' perspective, the three phases were independent of each other.

\section{Results}

Exposure duration at test The exposure duration at which subjects were tested was entered into a 2 (age group) $\times 2$ (spatial contiguity) ANOVA. Mean exposure durations in the different conditions were the following: contiguous-younger, $M=46.2 \mathrm{~ms}$; contiguous-older, $M=153.2 \mathrm{~ms}$; separatedyounger, $M=46.1 \mathrm{~ms}$; separated-older, $M=111.1 \mathrm{~ms}$. Analysis of exposure durations revealed a significant main effect of age group, $F(1,68)=76.83, p<.001$, indicating longer exposures in the older $(M=132.2 \mathrm{~ms})$ than in the younger $(M=46.1 \mathrm{~ms})$ subjects. There was also a significant main effect of spatial contiguity, $F(1,68)=4.62, p<.05$, with longer mean exposure duration in the contiguous condition $(M=99.7 \mathrm{~ms})$ than in the separated condition $(M=78.6 \mathrm{~ms})$. These main effects were qualified by a significant age group $\times$ spatial contiguity interaction, $F(1$, $68)=4.59, p<.05$, indicating a significant difference in exposure duration between the contiguous and the separated conditions in older subjects, $t(34)=2.33, p<.05$, but no difference in exposure durations between the contiguous and the separated conditions in younger subjects, $t<1$. Importantly, these different exposure durations yielded matched performance in the baseline (new) condition across groups as intended, as was evidenced by a 2 (age group) $\times 2$ (spatial contiguity) ANOVA, with all $p \mathrm{~s}>.30$.

Perceptual identification For each individual, the percentage of accuracy of perceptual identification in each condition was calculated. A trial was considered correct if both items were named accurately (or with sufficient specificity to ensure adequate perception). Mean accuracy across subjects in each age group for each condition is summarized in Fig. 4.

To assess associative priming, a 2 (age group: younger, older $) \times 2$ (spatial contiguity: contiguous, separated) $\times 2$ (pair type: old, recombined) mixed ANOVA was conducted. This analysis revealed a significant main effect of spatial contiguity, $F(1,68)=8.08, p<.01$, indicating better overall performance in the contiguous condition $(M=53.2 \%, S D=$ $14.8 \%)$ than in the separated condition $(M=44.9 \%, S D=$ $11.1 \%)$. We also found a significant main effect of pair type, $F(1,68)=50.14, p<.001$, with higher accuracy in the old condition $(M=66.4 \%, S D=16.9 \%)$ than in the recombined condition $(M=54.3 \%, S D=17.6 \%)$. A significant main effect of age was also found, $F(1,68)=$ $4.13, p=.05$, with older adults $(M=63.9 \%, S D=14.2 \%)$ outperforming younger adults $(M=56.8 \%, S D=16.3 \%)$ across the two pair types. Finally, a marginally significant pair type $\times$ spatial contiguity interaction was observed, $F(1$, $68)=2.98, p=.09$. Whereas significant associative priming was found in both the contiguous and the separated conditions, the magnitude of the effect was numerically larger in the contiguous condition (mean associative priming $=15.0 \%$ ) than in the separated condition (mean associative priming $=9.1 \%$ ). No other effects approached significance, with all $p \mathrm{~s}>.20 .{ }^{4}$ Importantly, the absence of an age group $\times$ pair type interaction suggested that associative priming effect was unaffected by age, and the absence of a three-way interaction indicated that this held both in the contiguous and in the separated conditions.

To assess item priming, a second 2 (age group) $\times 2$ (spatial contiguity) $\times 2$ (pair type: recombined, new) mixed ANOVA was conducted. This analysis revealed a significant main effect of pair type, $F(1,68)=225.54, p<.001$, with higher accuracy in the recombined condition than in the new condition $(M=$ $26.4 \%, S D=14.3 \%$ ). A marginally significant main effect of spatial contiguity was also found, $F(1,68)=3.53, p=.06$, indicating better overall performance in the contiguous

\footnotetext{
${ }^{4}$ As in Experiment 1, baseline (i.e., recombined) performance was not matched across conditions. To ensure that interpretation of the priming results was not compromised by different baseline performance across conditions, we analyzed results from a subset of subjects whose recombined performance was matched across groups and across spatial contiguity conditions (contiguous-younger, $n=17$, recombined $M=$ $53.1 \%$; contiguous-older, $n=14$, recombined $M=54.1 \%$; separatedyounger, $n=14$, recombined $M=53.9 \%$; separated-older, $n=16$, recombined $M=55.4 \%$ ). A 2 (age group) $\times 2$ (spatial contiguity) $\times 2$ (pair type: old, recombined) mixed ANOVA on their accuracy data revealed largely the same pattern as that obtained in the analysis of the full data set. There was a significant main effect of pair type, $F(1,57)=$ $37.89, p<.001$, confirming better performance in the old condition $(M=$ $65.8 \%, S D=15.6 \%)$ than in the recombined condition $(M=54.1 \%, S D=$ $15.5 \%)$. Critically, a significant pair type $\times$ spatial contiguity interaction was also observed, $F(1,57)=5.90, p<.05$, showing that although associative priming was significant in both spatial contiguity conditions (both $p \mathrm{~s}<.01$ ), the associative priming effect in the contiguous condition (mean associative priming $=16.0 \%$; old, $M=69.5 \%, S D=17.6 \%$ ) was significantly larger than that in the separated condition (mean associative priming $=7.2 \%$; old, $M=61.9 \%, S D=12.3 \%$ ). No effects involving age were significant.
} 
Fig. 4 Perceptual identification performance on old, recombined, and new trials in the contiguous and separated conditions for both age groups (Experiment 2)

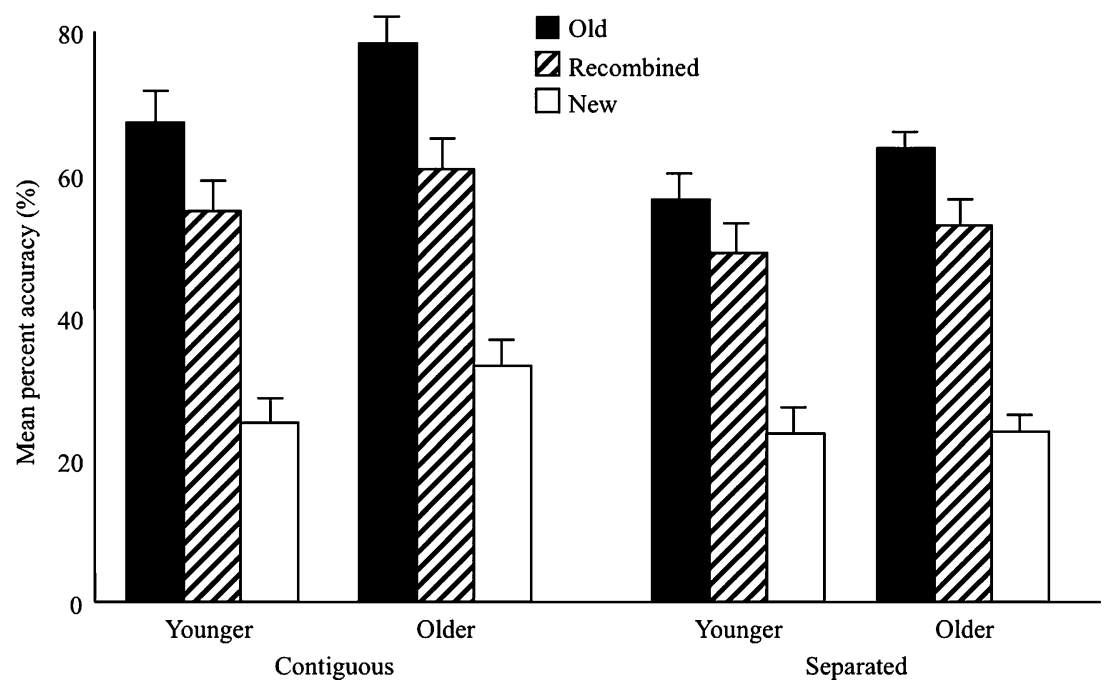

condition $(M=40.3 \%, S D=13.9 \%)$ than in the separated condition $(M=39.9 \%, S D=11.3 \%)$. No other effects approached significance, with all $p \mathrm{~s}>.15 .^{5}$

\section{Discussion}

In Experiment 2, we observed significant and comparable pictorial association priming in younger and older subjects, regardless of spatial contiguity. This finding contradicts the hypothesis that new association priming is spared in aging exclusively under conditions that promote perceptual unitization.

Data from the present experiment provide additional support for the notion that the observed associative effects are implicitly mediated. Because explicit memory is highly susceptible to aging (e.g., Naveh-Benjamin, 2000), the fact that associative priming is age invariant suggests that such priming in younger individuals is not inflated by explicit memory. In other words, if new association priming in pictures were mediated by explicit memory, we should have observed younger adults outperforming older adults.

Further evidence against the presence of explicit contamination comes from an examination of errors in the recombined condition (see also Experiment 1, Discussion section). As was described earlier, if individuals' performance were contaminated by explicit memory, we would expect to observe intrusions of the studied associates on incorrect recombined trials. Such errors, however, were very rare. In the contiguous condition, $6.2 \%$ of recombined

\footnotetext{
${ }^{5}$ Because baseline (i.e., new) performance was not matched across the spatial contiguity conditions, we repeated the ANOVA on the basis of data from a matched subset of subjects (contiguous-younger, $n=16$; contiguous-older, $n=15$; separated-younger, $n=16$; separated older, $n=16$ ) and found only the main effect of pair type (recombined > new) to be significant, $F(1,59)=191.53, p<.001$.
}

errors made by younger subjects were intrusions $(0.56$ intrusion errors out of 9.0), and the corresponding percentage for older adults was $1.4 \%$ ( 0.11 intrusion errors out of 7.8). In the separated condition, $3.8 \%$ of recombined errors made by younger subjects were intrusions ( 0.39 intrusion errors out of 10.2), and the corresponding percentage for older adults was $5.3 \%$ ( 0.50 intrusion errors out of 9.4).

One issue that deserves consideration is the fact that exposure durations differed considerably across age groups. Such exposure differences are to be expected, given ageassociated visual slowing (Schaie, 1989), and are necessary in order to compare priming across groups at similar accuracy levels. As was intended, these exposure conditions yielded equivalent accuracy across groups in the baseline (new) condition, but performance differences remained in the recombined condition. Therefore, to ensure that accuracy differences in the recombined condition did not contribute to age-invariant associative priming, we also compared subgroups of younger and older subjects whose performance in the recombined condition was equivalent (see Footnote 4). In both sets of comparisons (i.e., entire data set and subset of data), the magnitude of new association priming did not differ in younger and older subjects, suggesting that the reinstatement of a newly established association facilitates perceptual identification to a similar extent in younger and older subjects.

The findings from Experiment 2 suggest that pictorial association priming is mediated by a system that is resilient to aging, and one candidate system is the perceptual representation system (PRS). The PRS refers to a collection of domain-specific processing systems that are dedicated to the processing of perceptual information in different domains, such as words, objects, and auditory information (Tulving \& Schacter, 1990). As Mitchell (1993) suggested, the PRS is far less susceptible to the negative effects of aging than are other cognitive systems. Light et al. (1995), 
borrowing from Mandler (1980), have suggested that new association priming in aging is supported by the activation of novel representations, formed by the integration of perceptual elements that are "newly juxtaposed." This process of integration may underlie the formation of new representations in the PRS. The extended exposure durations required for identification of briefly presented stimuli in older subjects suggest that access to perceptual representations may take longer with age. However, the facilitatory effect of prior study on access to these representations is comparable to that seen in younger subjects. In Experiment 3, we sought further evidence for the notion that novel association priming for picture pairs is mediated by the PRS.

\section{Experiment 3: effects of stimulus format change on novel association priming}

The central findings from Experiments 1 and 2 are that novel association priming extends to pictorial stimuli and that such priming is modulated by spatial contiguity, but not by aging. The observed facilitation may be mediated by the PRS, a modality-specific system that is resilient to aging effects. Previous studies have established that perceptually mediated priming is sensitive to perceptual manipulations between study and test (e.g., Srinivas \& Roediger, 1990; ThompsonSchill \& Kan, 2001; Weldon, 1991). To test the perceptual basis of the observed associative priming, we altered the stimulus presentation format between study and test. Specifically, stimuli were presented as word pairs to be read aloud during the study phase and as picture pairs to be named during the test phase. We included only a separated study condition, because spatially separated presentation of the pictures was sufficient to elicit associative priming in Experiments 1 and 2 and the additional effect of spatial contiguity on associative priming was necessarily perceptual in nature. Thus, although the previous experiments revealed the role of perceptual unitization in pictorial association priming, they left unanswered the basic nature of the associative priming effect in the absence of perceptual unitization (i.e., in the spatially separate condition). If format change between study and test substantially reduces or eliminates priming, this would suggest a perceptual basis for associative priming, mediated by the PRS.

Method

\section{Subjects}

Twenty-three naïve college students were tested in the present experiment, in which the stimulus format was altered between study and test (i.e., changed-format condition). In order to compare their performance with that of the subjects in a same-format condition, data from 23 individuals who had participated in the separated condition in Experiments 1 and 2 were included. In accordance with the procedures of the Institutional Review Board, all individuals provided informed consent prior to testing. Subjects were paid for their time, and they were tested individually.

Since variability in perceptual speed was expected to be low among younger adults, we utilized the same exposure duration $(33 \mathrm{~ms})$ for all the subjects at perceptual identification, as in Experiment 1. Thus, we selected comparison subjects from Experiments 1 and 2 who were tested at an exposure duration of $33 \mathrm{~ms}$. In sum, 23 subjects from Experiment $1(n=18)$ and Experiment $2(n=5)$ who had participated in the separated condition were selected as comparison subjects, and 23 new subjects were tested in the present experiment.

Design and materials

The same stimuli were used as in the separated condition of Experiments 1 and 2, but these stimuli were presented in the form of verbal labels in the study phase and as pictures in the test phase. Pair type (old, new, recombined) was manipulated within subjects.

\section{Procedure}

As in Experiment 1, each experimental session consisted of three phases: practice, study (word reading), and test (perceptual identification of pictures). From the subjects' perspective, the three parts were independent of each other. All the stimuli were presented using PsychLab stimulus presentation software on a Macintosh PowerBook computer. Each session lasted approximately $25 \mathrm{~min}$.

Practice The practice phase was identical to that used in Experiment 1.

Study phase (word reading) Pairs of object names were presented in full view on the computer screen, one pair at a time, and subjects were asked to read the object names aloud. A total of 40 pairs of object names were presented, and each pair remained on the screen until a response was made.

Test phase (perceptual identification) The perceptual identification procedure was identical to that used in Experiment 1.

\section{Results}

For each individual, the percentage of accuracy of perceptual identification in each condition was calculated. A trial was considered correct if both items were named 
accurately (or with sufficient specificity to ensure adequate perception).

On average, subjects in the changed-format condition performed similarly across the three conditions (old condition, $M=25.5 \%, S D=12.1 \%$; recombined condition, $M=$ $23.9 \%, S D=12.2 \%$; new condition, $M=21.1 \%, S D=$ $12.9 \%$ ), as confirmed by a one-way ANOVA on percentage of accuracy, $F(2,44)=1.44, p=.25$. These data suggest that name reading does not promote pictorial associative (or item) priming.

To evaluate the effect of stimulus format change on associative priming and item priming, we compared performance in subjects from this experiment with that of a subset of subjects in the separated condition from Experiments 1 and 2 (see Fig. 5).

We assessed the effects of format change on associative priming with a two-way mixed factorial ANOVA with pair type (old, recombined) as the within-subjects factor and studytest format (same, changed) as the between-subjects factor. There was a main effect of pair type, $F(1,44)=8.44, p<.01$, indicating better performance in the old condition $(M=$ $39.7 \%, S D=19.7 \%$, than in the recombined condition $(M=$ $34.7 \%, S D=17.9 \%)$. There was also a significant main effect of format, $F(1,44)=43.48, p<.001$, revealing better overall performance in the same-format group $(M=49.6 \%, S D=$ $14.4 \%)$ than in the changed-format group $(M=20.9 \%, S D=$ $15.4 \%$ ). Importantly, these effects were modified by a marginally significant interaction between pair type and format, $F(1,44)=3.87, p=.06$, suggesting a larger associative priming effect in the same-format group (mean difference $=8.4 \%, t(22)=3.18, p<.05)$ than in the changedformat group (mean difference $=1.6 \%, t(22)<1$ ).

To evaluate item priming, we conducted a two-way mixed factorial ANOVA with pair type (recombined, new) as the within-subjects factor and format (same, changed) as the

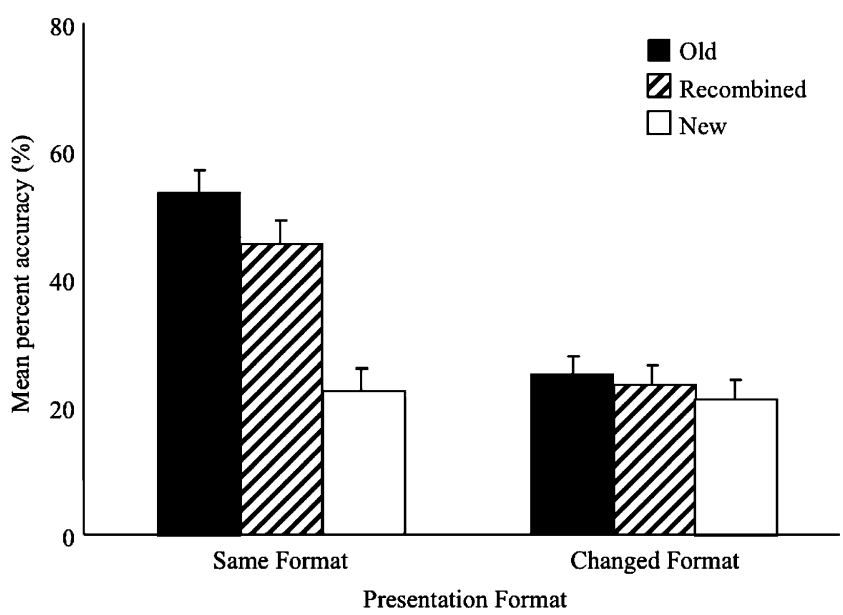

Fig. 5 Perceptual identification performance on old, recombined, and new trials in the same-format and changed-format conditions (Experiment 3) between-subjects factor. This analysis revealed a significant main effect of pair type, $F(1,44)=49.83, p<.001$, suggesting superior performance in the recombined condition than in the new condition $(M=21.9 \%, S D=13.6 \%)$. The main effect of format was also significant (same format, $M=34.0 \%, S D=$ $14.1 \%$; changed format, $M=22.5 \%, S D=10.9 \%), F(1,44)=$ $9.60, p<.01$. Critically, a significant interaction between pair type and format was found, $F(1,44)=30.29, p<.001$, revealing a significant item priming effect in the same-format group (mean difference $=22.8 \%$ ), $t(22)=9.09, p<.001$, and a nonsignificant item priming effect in the changed-format group $($ mean difference $=2.8 \%), t(22)=1.1, p=.29$.

\section{Discussion}

In Experiment 3, we found that a format change between study and test eliminated associative priming, consistent with the notion that the associative priming effects observed in Experiments 1 and 2 were mediated by a perceptual representation system. Although item priming was not the focus of the present study, the lack of item priming in the changed-format condition suggests that the item priming observed in Experiments 1 and 2 was also likely to have been mediated perceptually, consistent with a large body of evidence on item priming in the perceptual identification task (for a review, see Roediger \& McDermott, 1993).

Data from this experiment also speak to the issue of explicit contamination. If associative priming for pictures were driven by explicit memory, format change between study and test should exert little impact on the observed priming effect (Curran \& Schacter, 1996). Our finding of associative priming being eliminated as a result of format change is consistent with the idea that associative priming is supported by implicit memory (Graf \& Schacter, 1989).

\section{General discussion}

The present findings broaden our understanding of implicit memory for novel associations in several ways. First, our findings of reliable associative priming in the pictorial domain indicate that it is possible to obtain associative priming under conditions that do not entail the creation of a new emergent conceptual representation. Second, we demonstrate that such priming is not dependent on spatial contiguity between the component pictures, arguing that perceptual unitization is not a necessary condition for novel pictorial association priming. Nevertheless, priming is enhanced by conditions that promote perceptual unitization. Third, to our knowledge, our study provides the first example of significant associative priming for pictures in older adults and demonstrates that the magnitude of priming is similar to that obtained in younger subjects, regardless of spatial contiguity. The latter finding argues 
against the notion that intact associative priming in aging is limited to conditions that foster unitization.

In addition to further delineating the conditions under which novel association priming emerges, our data also inform our understanding of the mechanisms that underlie implicit memory for novel associations in aging. Consistent with the idea that priming for novel pictorial associations is perceptually mediated, we found in Experiment 3 that format change between study and test disrupted such priming. Light et al. (1995) have suggested that new association priming in aging may reflect the formation of novel perceptual representations that integrate disparate perceptual elements. The PRS is a likely system to support such representations. Mitchell (1993) suggested that the PRS is "hard-wired from the start and [is] very hardy in surviving the effects of aging" (p. 173). Our results add to a growing literature suggesting that implicit memory is immune to the negative effects of aging (for reviews, see Light et al., 2000; Mitchell, 1993; Mitchell \& Bruss, 2003; Parkin, 1993).

The finding of significant associative priming in the separated condition suggests that the formation of novel representations in the PRS is not dependent on spatial contiguity but, rather, can be supported by simultaneous presentation of two stimuli, even when they are separated in space. We postulate that spatial contiguity promotes perceptual unitization of the component stimuli (Treisman et al., 1983), but it is possible that even the separated condition may have permitted some degree of perceptual unitization, since the two objects were photographed against a uniform background. We conducted a follow-up experiment in which a 1-in.-wide white space was introduced between the two objects in an object pair, such that the two objects were no longer against a continuous background. In a group of naïve younger subjects $(n=18)$, we found significant associative priming in this different-background condition (old, $M=$ $64.4 \%, S D=16.1 \%$; recombined, $M=53.2 \%, S D=$ $13.5 \%), t(17)=2.77, p=.01$. Critically, the magnitude of associative priming did not differ between the two separated conditions (comparison data for the same background separated condition were drawn from Experiment 2), $F(1,34)<1$. These data suggest that perceptual unitization, promoted either by spatial contiguity of objects or by continuity of background, is not necessary for associative priming.

In sum, our findings provide evidence that implicit memory for novel associations can occur in the absence of an emergent conceptual representation. Novel pictorial association priming is enhanced by, but does not require, the establishment of a perceptually unitized representation, and such priming is age invariant. The demonstration that implicit memory can support the establishment of novel associations between pictures extends the boundaries of implicit memory. Additionally, our findings shed light on the conditions under which new association priming is intact in aging.

\section{References}

Ceraso, J. (1985). Unit formation in perception and memory. In G. H. Bower (Ed.), The psychology of learning and motivation, vol 19 (pp. 179-210). Orlando: Academic Press.

Chun, M. M., \& Phelps, E. A. (1999). Memory deficits for implicit contextual information in amnesic subjects with hippocampal damage. Nature Neuroscience, 2, 844-847.

Curran, T., \& Schacter, D. L. (1996). Implicit memory and perceptual brain mechanisms. In D. Hermann, C. L. McEvoy, C. Hertzog, P. Hertel, \& M. K. Johnson (Eds.), Basic and applied memory research: Theory in context, vol 1 (pp. 221-240). Mahwah: Erlbaum.

Dean, M. P., \& Young, A. W. (1996). An item-specific locus of repetition priming. Quarterly Journal of Experimental Psychology, 49A, 269-294.

Ebbinghaus, H. (1885). Memory: A contribution to experimental psychology (H. A. Ruger \& C. E. Bussenues, Trans.). New York: Columbia University, Teachers College.

Fleischman, D. A., \& Gabrieli, J. D. E. (1998). Repetition priming in normal aging and Alzheimer's disease: a review of findings and theories. Psychology and Aging, 13, 88-119.

Gabrieli, J. D. E., Keane, M. M., Zarella, M. M., \& Poldrack, R. A. (1997). Preservation of implicit memory for new associations in global amnesia. Psychological Science, 8, 326-329.

Goshen-Gottstein, Y., \& Moscovitch, M. (1995). Repetition priming effects for newly formed associations are perceptually based: evidence from shallow encoding and format specificity. Journal of Experimental Psychology. Learning, Memory, and Cognition, 21, 1249-1262.

Goshen-Gottstein, Y., Moscovitch, M., \& Melo, B. (2000). Intact implicit memory for newly formed verbal associations in amnesic patients following single study trials. Neuropsychology, 14, 570578.

Graf, P., \& Schacter, D. L. (1985). Implicit and explicit memory for new associations in normal and amnesic subjects. Journal of Experimental Psychology. Learning, Memory, and Cognition, 11, 501-518.

Graf, P., \& Schacter, D. L. (1989). Unitization and grouping mediate dissociations in memory for new associations. Journal of Experimental Psychology. Learning, Memory, and Cognition, 15, 930-940.

Howard, D. V., Heisey, J. G., \& Shaw, R. J. (1986). Aging and the priming of newly learned associations. Developmental Psychology, 22, 78-85.

Howard, D. V., Fry, A. F., \& Brune, C. M. (1991). Aging and memory for new associations: direct versus indirect measures. Journal of Experimental Psychology. Learning, Memory, and Cognition, 17, 779-792.

Keane, M. M., \& Verfaellie, M. (2003). Amnesia: Cognitive neuropsychological issues. In T. E. Feinberg \& M. J. Farah (Eds.), Behavioral neurology and neuropsychology (2nd ed., pp. 445-456). New York: McGraw-Hill.

Keane, M. M., Gabrieli, J. D. E., Noland, J. S., \& McNealy, S. I. (1995). Normal perceptual priming of orthographically illegal nonwords in amnesia. Journal of the International Neuropsychological Society, 1, 425-433.

La Voie, D., \& Light, L. L. (1994). Adult age differences in repetition priming: a meta-analysis. Psychology and Aging, 9, 539-553. 
Light, L. L., Kennison, R., Prull, M. W., La Voie, D., \& Zuellig, A. (1996). One-trial associative priming of nonwords in young and older adults. Psychology and Aging, 11, 417-430.

Light, L. L., La Voie, D., \& Kennison, R. (1995). Repetition priming of nonwords in young and older adults. Journal of Experimental Psychology. Learning, Memory, and Cognition, 21, 327-346.

Light, L. L., Prull, M. W., La Voie, D., \& Healy, M. R. (2000). Dualprocess theories of memory in old age. In T. J. Perfect \& E. A. Maylor (Eds.), Models of cognitive aging (pp. 238-300). New York: Oxford University Press.

Mandler, G. (1980). Reocgnizing: the judgment of previous occurrence. Psychological Review, 87, 252-271.

Mandler, G. (1982). The integration and elaboration of memory structures. In F. Klix, J. Hoffman, \& E. van der Meer (Eds.), Cognitive research in psychology (pp. 33-40). Amersterdam: North-Holland.

Mattis, S. (2008). Dementia rating scale-2 (DRS-2). Odessa: Psychological Assessment Resources.

Mayes, A. R., Montaldi, D., \& Migo, E. (2007). Associtive memory and the medial temporal lobes. Trends in Cognitive Sciences, 11, $126-135$.

Micco, A., \& Masson, M. E. J. (1991). Implicit memory for new associations: an interactive process approach. Journal of Experimental Psychology. Learning, Memory, and Cognition, 17, $1105-1123$.

Mitchell, D. B. (1993). Implicit and explicit memory for pictures: Multiple views across the lifespan. In P. Graf \& M. E. J. Masson (Eds.), Implicit memory: New directions in cognition, development, and neuropsychology (pp. 171-190). London: Erlbaum.

Mitchell, D. B., \& Bruss, P. J. (2003). Age differences in implicit memory: conceptual, perceptual, or methodological? Psychology and Aging, 18, 807-822.

Moscovitch, M., Winocur, G., \& McLachlan, D. (1986). Memory as assessed by recognition and reading time in normal and memoryimpaired people with Alzheimer's disease and other neurological disorders. Journal of Experimental Psychology: General, 115, 331-347.

Musen, G., \& Squire, L. R. (1993a). Implicit learning of color-word associations using a Stroop paradigm. Journal of Experimental Psychology. Learning, Memory, and Cognition, 19, 789-798.

Musen, G., \& Squire, L. R. (1993b). On the implicit learning of novel associations by amnesic patients and normal subjects. Neuropsychology, 7, 119-135.

Musen, G., Szerlip, J. S., \& Szerlip, N. J. (1999). Role of familiarity and unitization on new-association priming. Journal of Experimental Psychology: Learning, Memory \& Cognition, 25, 275-283.

Naveh-Benjamin, M. (2000). Adult age differences in memory performance: tests of an associative deficit hypothesis. Journal of Experimental Psychology. Learning, Memory, and Cognition, $26,1170-1187$.

Old, S. R., \& Naveh-Benjamin, M. (2008). Memory for people and their actions: further evidence for an age-related associative deficit. Psychology and Aging, 23, 467-472.

Paller, K. A., \& Mayes, A. R. (1994). New-association priming of word identification in normal and amnesic subjects. Cortex, 30, 53-73.

Parkin, A. J. (1993). Implicit memory across the lifespan. In P. Graf \& M. E. J. Masson (Eds.), Implicit memory: New directions in cognition, development, and neuropsychology (pp. 191-206). London: Erlbaum.

Pecher, D., \& Raaijmakers, J. G. W. (2004). Priming for new associations in animacy decision: evidence for context depen- dency. The Quarterly Journal of Experimental Psychology, 57, 1211-1231.

Quamme, J. R., Yonelinas, A. P., \& Norman, K. A. (2007). Effect of unitization on associative recognition in amnesia. Hippocampus, 17, 192-200.

Reingold, E. M., \& Goshen-Gottstein, Y. (1996). Separating consciously controlled and automatic influences in memory for new associations. Journal of Experimental Psychology. Learning, Memory, and Cognition, 22, 397-406.

Rhodes, M. G., Castel, A. D., \& Jacoby, L. L. (2008). Associative recognition of face pairs by younger and older adults: the role of familiarity-based processing. Psychology and Aging, 23, 239249.

Roediger, H. L., III, \& McDermott, K. B. (1993). Implicit memory in normal human subjects. In H. Spinnler \& F. Boller (Eds.), Handbook of neuropsychology, vol 8 (pp. 63-131). Amsterdam: Elsevier.

Sadoski, M., \& Paivio, A. (2001). Imagery and text: A dual coding theory of reading and writing. Mahwah: Erlbaum.

Schacter, D. L., \& Graf, P. (1986). Effects of elaborative processing on implicit and explicit memory for new associations. Journal of Experimental Psychology. Learning, Memory, and Cognition, 12, $432-444$.

Schaie, K. W. (1989). Perceptual speed in adulthood: cross-sectional and longitudinal studies. Psychology and Aging, 4, 443-453.

Srinivas, K., \& Roediger, H. L., III. (1990). Classifying implicit memory tests: category association and anagram solution. Journal of Memory and Language, 29, 389-412.

Thompson-Schill, S. L., \& Kan, I. P. (2001). Perceptual and conceptual sources of priming on a word generation task. Memory \& Cognition, 29, 698-706.

Treisman, A., Kahneman, D., \& Burkell, J. (1983). Perceptual objects and the cost of filtering. Perception \& Psychophysics, 33, 527 532.

Tulving, E., \& Schacter, D. L. (1990). Priming and human memory systems. Science, 247, 301-306.

Uttl, B., Graf, P., \& Cosentino, S. (2003). Implicit memory for new associations: Types of conceptual representations. In J. S. Bowers \& C. J. Marsolek (Eds.), Rethinking implicit memory (pp. 302 323). New York: Oxford University Press.

Weldon, M. S. (1991). Mechanisms underlying priming on perceptual tests. Journal of Experimental Psychology. Learning, Memory, and Cognition, 17, 526-541.

Yang, J., Weng, X., Guan, L., Kuang, P., Zhang, M., Sun, W., et al. (2003). Involvement of the medial temporal lobe in priming for new associations. Neuropsychologia, 41, 818-829.

Zeelenberg, R., Pecher, D., \& Raaijmakers, J. G. W. (2003). Associative repetition priming: A selective review and theoretical implications. In J. S. Bowers \& C. J. Marsolek (Eds.), Rethinking implicit memory (pp. 261-283). New York: Oxford University Press.

\section{Authors' Note}

This research was supported by National Institutes of Health Grants MH 57681 and MH 070830 and by the Medical Research Service of the Department of Veterans Affairs. We thank Amanda Utevsky for her assistance with data coding, and we also thank the reviewers for their helpful comments on an earlier draft of the manuscript. 